\title{
Dart Dosing Unit
}

National Cancer Institute

\section{Source}

National Cancer Institute. Dart Dosing Unit. NCI Thesaurus. Code C149426.

A unit of presentation used to represent the quantity of product that is found in a single dart device. 